\title{
Hemorrhagic Auricular Schwannoma: A case report
}

\section{Case \\ Report}

\author{
Raid M. Al-Ani
}

Department of Surgery (Otolaryngology), College of Medicine, University of Anbar, Anbar, Iraq

\begin{abstract}
Introduction: Schwannoma is a benign tumor of Schwann cells and is seldom to be seen in the auricle. In addition to the rarity of auricular schwannoma (AS), intratumoral bleeding is never previously reported.

Case report: A 35-year-old female patient who has presented with right painless auricular mass for 2 years. A sudden and rapid increase in its size occurred in the last 2 months before presentation. The mass was excised under general anesthesia. Histopathological examination proved the diagnosis of AS.

Discussion: Schwannoma is a slow-growing benign tumor. It commonly affects the head and neck region between 25 $45 \%$. And vestibular schwannoma is the commonest type of schwannomas in this area. The first case of AS was reported by Fodor et al. in 1977. Since that time only 4 cases were reported in the world. Bleeding within schwannomas e.g. vestibular schwannoma was reported, but it is not reported in case of AS. Therefore, this hemorrhagic AS was the first reported case in the world.

Conclusion: AS is seldom seen in clinical practice. And intratumoral bleeding is never reported before.
\end{abstract}

Key Words: Auricle, ear, external ear canal, pinna, schwannoma.

Received: $27^{\text {th }}$ June 2019, Accepted: $07^{\text {th }}$ September 2019

Corresponding Author: Raid M. Al-Ani, Department of Surgery (Otolaryngology), College of Medicine, University of Anbar, Anbar, Iraq, Tel.: 009647906145364, E-mail: raedalani2003@yahoo.com

ISSN: 2090-0740, March 2020 Vol. 21, No. 1.

\section{INTRODUCTION}

Schwannomas are slowly growing benign tumor of neuro-ectodermal origin. Schwannomas are well known to arise from Schwann cell of the branches of peripheral, cranial or autonomic nerves. They are usually presented as a painless solitary swelling. They are affecting the head and neck in a $25-45 \%$, where the vestibular schwannoma is the commonest. The presentation of head and neck schwannomas depends on their location. The auricle is a rare site of affection by schwannoma ${ }^{[1]}$. The first case of external ear schwannoma was reported in $1977^{[2]}$. When we were reviewing the kinds of literature, only five cases of auricular schwannomas were reported in the world ${ }^{[1-5]}$. All of them are presented without bleeding within the tumors. In the present article, we describe a further case of auricular schwannoma and the first case of hemorrhagic AS.

\section{CASE REPORT}

A 35-year-old female was presented to the outpatient Otolaryngology clinic in AL-Hussein Teaching Hospital/ Samawah city with painless swelling over the posterior aspect of the right auricle (Figure 1) 2 years ago. The swelling was gradually increasing in size until the last 2 months before the presentation when there was a sudden increment in its size (2.5 times than the prior one). There was no history of previous trauma or surgery. No other ear, nose, and throat or general symptoms.

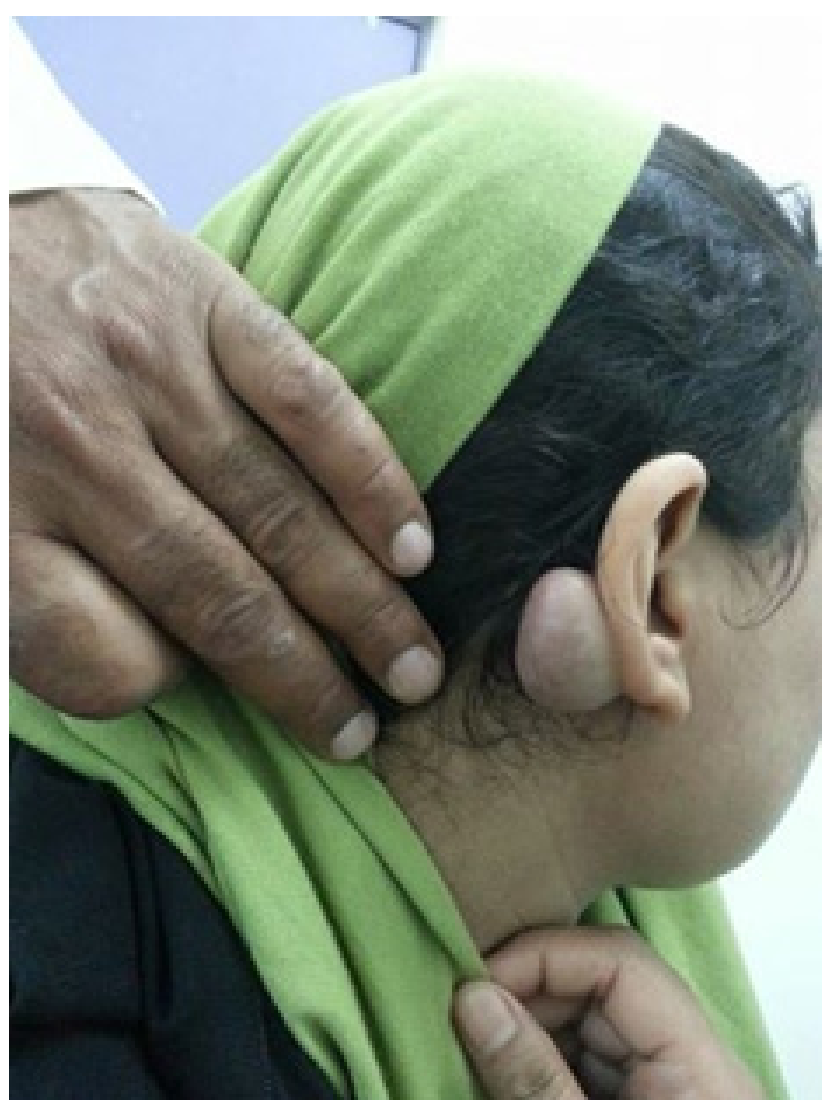

Fig. 1: The patient with right auricular mass 
The mass was non-tender, oval in shape, measured $5 \times 3$ $\mathrm{cm}$. in diameters, freely mobile, fluctuant and the overlying skin was warm on touch and there was an increased in its vascularity. There was no scar and no changes over the skin surrounding the swelling. The swelling was neither pulsatile nor compressible. Other ear, nose, and throat examination were completely normal. We put the sebaceous cyst, epidermoid cyst, lipoma, haemangioma, and keloid as a differential diagnosis for such mass. We decided to subject the patient for excisional biopsy without proceeding to radiological or fine-needle aspiration cytology owing to the easy accessibility of the mass.

The mass was easily excised completely under general anesthesia and sent for histopathological examination. Grossly the mass was an oval in shape, $5 \times 3 \mathrm{~cm}$ in diameters, when we excised part of it, its cavity contains blood with a thick wall but it contains no hair (Figure 2).

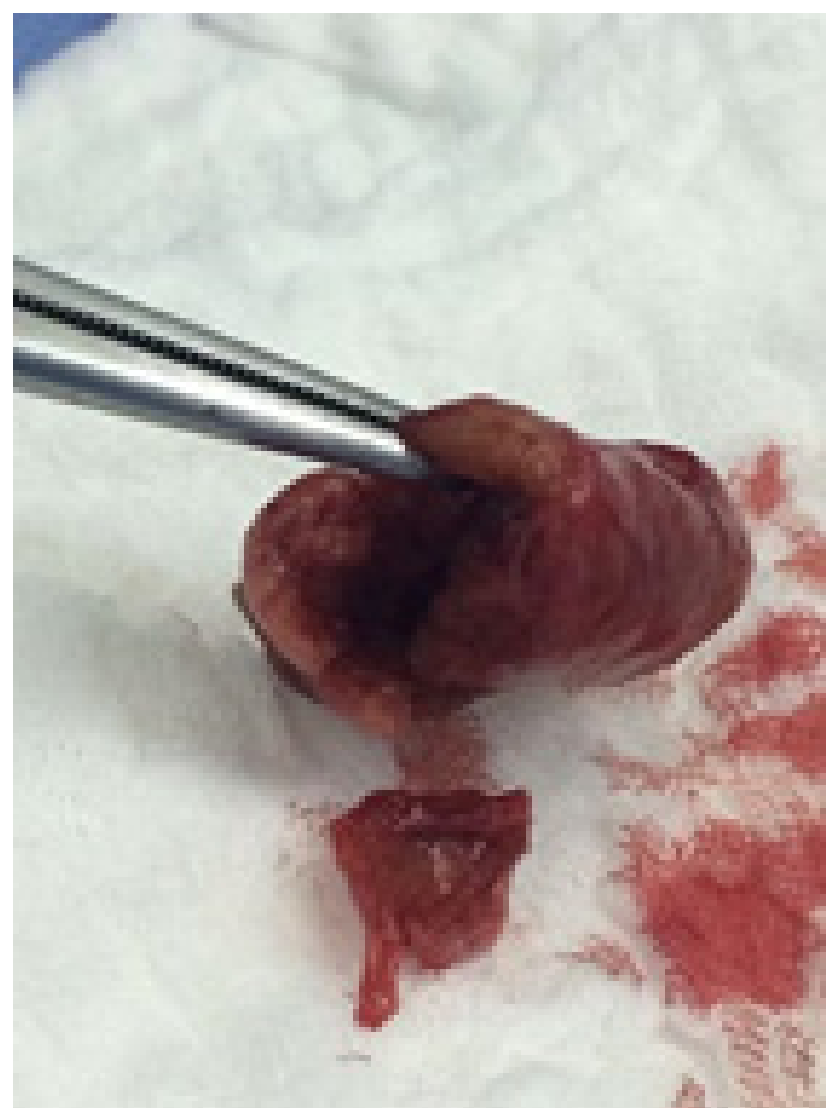

Fig. 2: The excised mass contains a blood with in its cavity and has a thick wall

The histopathological result showed the diagnosis of AS (Figure 3). The tumor cells were stained strongly for S-100 protein on immunohistochemical staining. The final diagnosis of the mass was established as AS. There is no recurrence during a 3 years follow-up. This study was approved by the scientific team of AL-Hussein Teaching Hospital in Sammawah city/Iraq.

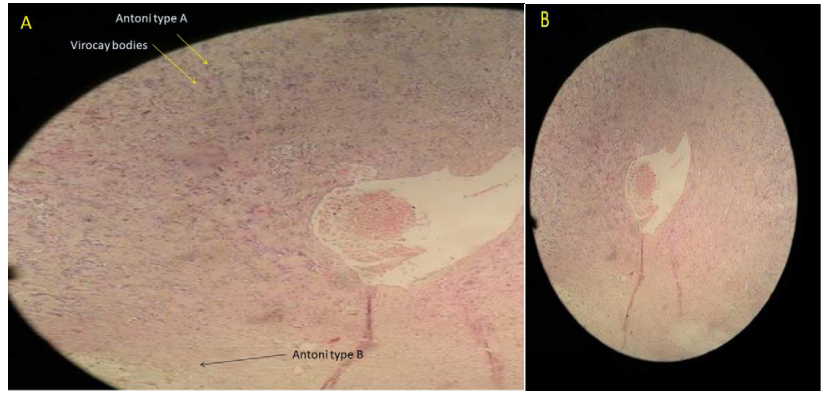

Fig. 3: Microscopic section of the tumor showing areas of compact spindle cells arrayed in a palisade pattern known as Antoni type A, Antoni type B, Verocay bodies and an area of hemorrhage within a cavity. (H\&E staining, A: 40× and B: 4× magnification power)

\section{DISCUSSION}

Verocay in 1908 was the first who described the solitary schwannoma and gave it the name of neurinoma; the name schwannoma was assigned by Batsakis in 1974. Schwannoma is also known by other terms, such as neurinoma, neurilemmoma, mioschwannoma, schwannoglioma, etc ${ }^{[6]}$. The first case of AS was reported by Fodor et al in 1977. Following this case, only 4 cases were reported in the world ${ }^{[1-5]}$.

Schwannoma is a slowly growing, painless, benign, encapsulated tumor arising from Schwann cell, so any nerves could be affected by this kind of tumor except the olfactory and optic nerves. The affection of the external ear by schwannoma is extremely rare ${ }^{[7]}$.

The nerve supply to the auricle is derived from auriculotemporal, greater auricular, lesser occipital and partly from facial and vagus nerves ${ }^{[1]}$. Owing to the location of the presenting case, the swelling may have originated from the branch of the greater auricular nerve.

Due to its rarity occurrence, AS is rarely put in the differential diagnosis of the swelling of the pinna. The final diagnosis of the schwannoma depends on histopathological evaluation and immunohistochemical study. The treatment of choice for such tumor is by complete surgical excision. The recurrence is rare after complete surgical removal ${ }^{[7]}$.

The presenting case has differed from the prior 5 reported $\operatorname{cases}^{[1-5]}$ in 1 . the size of the mass was larger, this was due to bleeding inside the mass creating a cavity within it. Therefore, the color of the swelling was changed in dusky color, and on physical examination, there was an increase in the skin temperature overlying the swelling with regards to the surrounded skin. It is well known that schwannoma (like vestibular schwannoma) might bleed causing a rapid increment in its size with newly developed symptoms due to pressure effects on the adjacent structures. The vestibular schwannoma is the commonest one of schwannomas in the body. However, intratumoral bleeding 
is extremely rare with a rate of $0.4 \%$ from all newly diagnosed vestibular schwannomas ${ }^{[8]}$. Micro-hemorrhages and vascular abnormalities might be the possible causes of such bleeding ${ }^{[9]}$. To our best knowledge, the presenting case was the first case in the world in which bleeding inside the tumor creates a cavity and causing rapid growth of the tumor. 2. The location was on the dorsal aspect of the auricle, this supports that schwannoma could affect any part of the auricle owing to its rich nerve supply.

\section{CONCLUSION}

Hemorrhagic AS of the presenting case was the first case reported in the world. Although, AS is a slowgrowing tumor, the sudden and rapid growth of it is due to hemorrhage inside the tumor.

\section{CONFLICT OF INTEREST}

There are no conflict of interest.

\section{REFERENCES}

1. Thakur K, Sharma SK, Raina R, Gupta VD. Auricular schwannoma: a rare presentation. Jaypee journals. 2013;10003:166-8.

2. Fodor RI, Pastore PN, Frable MA. Neurilemmoma of the auricle: a case report. Laryngoscope. 1977;87(10):1760-4.
3. Xing S, LÜ H. A case report of auricular neurilemmoma. Chinese J Otorhinolaryngol Head Neck Surg. 2008;43(6):450.

4. Lai JC, Tsai YL. Schwannoma of the auricle. B-ENT. 2010;6(4):281-3.

5. Lee DH, Kang HS, Park HJ. Schwannoma of the auricle. Otol Neurotol. 2012;33(9):e85-6.

6. Morais D, Santos J, Alonso M, Ovelar Y. Schwannoma of the external auditory canal: an exceptional location. Acta Otorrinolaringol (English Ed. 2007;58(4): 169-70.

7. Bennani A, Karich N, Kamaoui I, Chraibi M, Abbaoui S. Schwannoma with chondroid metaplasia of the external auditory canal-a rare finding in a rare location: a case report. J Med Case Rep. 2018;12(1):66.

8. Carlson ML, Tombers NM, Driscoll CLW, Van Gompel JJ, Lane JI, Raghunathan A, et al. Clinically significant intratumoral hemorrhage in patients with vestibular schwannoma. Laryngoscope. 2017;127(6):1420-6.

9. Mathkour M, Helbig B, McCormack E, Amenta PS. Acute Presentation of Vestibular Schwannoma Secondary to Intratumoral Hemorrhage: A Case Report and Literature Review. World Neurosurg. 2019. 